\title{
Weight changes and lifestyle behaviors in women after breast cancer diagnosis: a cross-sectional study
}

\author{
Yong Heng Yaw ${ }^{1}$, Zalilah Mohd Shariff, ${ }^{1}{ }^{*}$, Mirnalini Kandiah', Chan Yoke Mun ${ }^{1}$, Rokiah Mohd Yusof ${ }^{1}$, \\ Zabedah Othman ${ }^{3}$, Nurfaizah Saibul', Yong Heng Weay ${ }^{4}$ and Zailina Hashim ${ }^{4}$
}

\begin{abstract}
Background: Weight gain rather than weight loss often occurs after breast cancer diagnosis despite breast cancer survivors frequently reported making healthful lifestyle changes. This study describes the prevalence and magnitude of changes in weight before and after breast cancer diagnosis and examines lifestyle behaviors of breast cancer survivors with stable weight, weight gain or weight loss.

Methods: Respondents were 368 women with breast cancer characterized by stages I, II and III. All were recruited from hospitals or breast cancer support groups and had completed conventional treatment. Current weight and height were measured while weight at cancer diagnosis and 1 year before diagnosis were self-reported. Weight change was calculated as the difference between current weight and weight a year preceding breast cancer diagnosis. A 24-hour diet recall and Global Physical Activity Questionnaire assessed dietary intake and physical activity, respectively. Differences in lifestyle behaviors among weight change groups were examined using Analysis of Covariance (ANCOVA).
\end{abstract}

Results: Mean weight change from a year preceding diagnosis to study entry was $2.73 \mathrm{~kg}$ (95\% Cl: 1.90-3.55). Most women (63.3\%) experienced weight gain rather than weight loss (36.7\%) with a higher percentage (47.8\%) having at least $5 \%$ weight gain (47.8\%) rather than weight loss (22\%), respectively. Compared to other weight change groups, women in $>10 \%$ weight gain group had the lowest fruit and vegetable servings (1.58 servings/day; $95 \% \mathrm{Cl}$ : 1.36-1.82) and highest servings of dairy products (0.41 servings/day; $95 \% \mathrm{Cl}: 0.30-0.52$ ).

Conclusions: Weight gain was evident in this sample of women after breast cancer diagnosis. Information on magnitude of weight change after breast cancer diagnosis and lifestyle behaviors of breast cancer survivors with varying degrees of weight change could facilitate the development and targeting of effective intervention strategies to achieve healthy weight and optimal health for better survival.

\section{Background}

Breast cancer is the second most frequent cancer in the world, with an estimated 1.05 million cases in the year 2000 [1]. Breast cancer is also the most commonly diagnosed cancer among Malaysian women. Although the incidence of breast cancer in Malaysia is much lower compared to European countries, it is increasing steadily as it comprised $30.4 \%$ of all female cancers [2]. In 2006,

\footnotetext{
* Correspondence: zalilah@medic.upm.edu.my

'Department of Nutrition and Dietetics, Faculty of Medicine and Health

Sciences, Universiti Putra Malaysia, Serdang 43400 Selangor, Malaysia

Full list of author information is available at the end of the article
}

3,525 new cases of breast cancer were reported to the National Cancer Registry (NCR) of Malaysia, giving an age-standardized incidence rate of 39.3 per 100,000 women [3]. The 5-year survival of European women diagnosed in the period 1990-1994 was 77\% [4]. A higher figure of $90 \%$ was reported in the United States [5]. However, the overall 5-year survival from breast cancer reported for Malaysian women was $58 \%$ between 1993 and 1997 [6].

Weight change occurs in most women after breast cancer diagnosis and the amount of weight gain or loss could vary among the survivors [7]. Most cancers cause

\section{() Biomed Central}

(c) 2011 Yaw et al; licensee BioMed Central Ltd. This is an Open Access article distributed under the terms of the Creative Commons Attribution License (http://creativecommons.org/licenses/by/2.0), which permits unrestricted use, distribution, and reproduction in any medium, provided the original work is properly cited. 
weight loss, changes in appetite and dietary intake, reduced physical activity and deterioration of psychological well being. However, weight gain rather than weight loss has been frequently reported in patients after diagnosis of breast cancer. Halbert et al., (2008) [8] found that $47 \%$ of women reported weight gain, $32 \%$ weight loss and $21 \%$ no weight change. Studies have reported a weight gain ranging from 1.0 to $6.0 \mathrm{~kg}$ during the first year after being diagnosed with breast cancer [9-11]. However, greater gains are not uncommon [12].

Lifestyle change is one of the many possible actions women may take when they have been diagnosed with breast cancer in order to improve breast cancer prognosis and reduce the probability of cancer recurrence. Several studies [13-15] have found that breast cancer survivors changed their diets with the most common dietary changes reported were decreased consumption of dietary fat and increased intake of fruits and vegetables. Lower energy and macronutrient intakes post-diagnosis have also been reported in response to breast cancer diagnosis [16]. Besides making healthful dietary changes, breast cancer survivors were also more likely to increase or maintain their moderate to vigorous physical activity post-diagnosis [17-19].

As many developing countries, including Malaysia, have disproportionately high rates of breast cancer morbidity and mortality, a comprehensive assessment of lifestyle behaviors after breast cancer is essential as these behaviors may potentially affect prognosis, cancer recurrence, long-term survival and risk of other chronic diseases such as cardiovascular, hypertension and diabetes [11]. To date, information on lifestyle behaviors after breast cancer diagnosis is mainly derived from studies in western population. As lifestyle changes in response to cancer diagnosis could be influenced by various factors within specific settings, the lifetysle behaviors observed in the developing countries may differ from those reported in western population. Cancer awareness and knowledge, motivation to change, professional and social support, access to healthy food are among factors that may vary by cultures which could potentially influence lifestyle behaviors. This study reports on the prevalence and magnitude of weight changes after breast cancer diagnosis and examines self reported dietary intake and physical activity of breast cancer survivors with various levels of weight change.

\section{Methods}

\section{Study respondents}

This cross-sectional survey was conducted in eight general hospitals and four breast cancer support groups in seven states of Peninsular Malaysia. These states represented the Northern, Central, Southern and East Coast regions of Peninsular Malaysia. Respondents were recruited from oncology or surgical out-patient clinics of general hospitals and breast cancer support groups over a period of 10 months (February to December 2008). Lists of cancer patients attending the out-patient clinics for medical check-up as well as active members of support groups were provided by the hospitals and support groups, respectively. Out of 615 breast cancer survivors screened, 400 women met the selection criteria and were invited to participate in this study. A total of 368 women voluntarily participated in the study, giving a response rate of $92 \%$.

This study employed purposive sampling as the selection of respondents was based on several criteria i.e. women 20-65 years old at the time of diagnosis, diagnosed with cancer of stages 0-III, completed conventional treatments (surgery, chemotherapy, radiotherapy) at least 6 months prior to recruitment, free from cancer recurrence and not currently under any medication (e.g. hormonal pills) that would affect the study outcome. All respondents were interviewed using a pre-tested questionnaire either at the hospitals or premises of breast cancer support groups. The study protocol was approved by the Medical Research Ethics Committee of the Faculty of Medicine and Health Sciences, Universiti Putra Malaysia and the Medical Research Ethics Committee (MREC), Ministry of Health Malaysia. Prior to data collection, informed consent was obtained from all respondents.

\section{Measurements \\ Dietary intake}

A 24-hour recall was used to assess the dietary intakes of the respondents. Portion size, brand names and cooking method were recorded. Standard household measuring cups and spoons were used to assist respondents in portion size estimation. The nutritionist Pro $^{\mathrm{TM}}$ software version 2.5 (First Data Bank, USA, 2005) [20] was utilized to analyse the dietary data. Several food databases such as Malaysian Food Composition Tables [21], Singapore Food Facts (1999) [22], ASEAN Food Composition Tables [23] and USDA Food Databases were utilized in addition to those available in nutritionist Pro $^{\mathrm{TM}}$. For dishes that were not available in any food database, 2-3 recipes were used to calculate the energy and nutrient content, and the average values were used in dietary analysis. Total energy intake, percentage of energy from macronutrients and the number of servings for grains, meats and legumes, fruits, vegetables and dairy products were calculated.

\section{Physical activity}

The current physical activity level was measured using the Global Physical Activity Questionnaire (GPAQ) [24]. Women were requested to recall the number of days in the last seven days that they did vigorous intensity 
activity or/and moderate intensity activity at three major settings (activities at work/home, travel to and from places, recreational activities), as well as the number of hours and minutes per day they did the activities, respectively. The respective total hours for physical activity were calculated and multiplied with the metabolic equivalent (MET) hours per week and the values were then categorized into low, moderate and high.

\section{Anthropometric measurements}

The weight a year preceding breast cancer diagnosis, was based on self-reported information. All respondents indicated that the reported weights were stable for at least 6 months prior to diagnosis. Current weight and height were measured to the nearest $0.1 \mathrm{~kg}$ and $0.1 \mathrm{~cm}$, respectively using TANITA digital weighing scale (TANITA Corporation of America, Inc, United States) and SECA body meter (SECA, British Indicators Ltd, United Kingdom). Body mass index (BMI) was calculated and classified according to the World Health Organization classification [25]. Waist circumference was assessed using a SECA microtoise tape (SECA, British Indicators Ltd, United Kingdom). Waist circumference $\geq 80 \mathrm{~cm}$ for women was considered as at high risk for abdominal obesity [25].

Weight change was defined as the difference between the present weight and the weight a year preceding breast cancer diagnosis. Percent of weight change was calculated as $=$ [present weight $(\mathrm{kg})$ - weight a year preceding breast cancer diagnosis $(\mathrm{kg})] /$ weight a year preceding breast cancer diagnosis $(\mathrm{kg}) \mathrm{x} 100 \%$. The changes in weight for the women were then categorized into $>10 \%$ weight loss, $5-10 \%$ weight loss, no change $(<5 \%$ weight loss and weight gain), 5-10\% weight gain and $>10 \%$ weight gain groups [26]. These weight change categories were chosen as they are commonly used for weight loss recommendation to reduce the risk of obesity and cancer. A reduction of $5-10 \%$ of body weight is likely to have a significant beneficial health effect on those who need to lose weight, even if the ideal weight was not achieved.

\section{Other variables}

Information were also collected on socio-demographic background (e.g. age, education level, employment status, marital status), reproductive and breast cancer history (e.g. time since diagnosis, menopausal status, type of treatments), family history of breast and other specific cancers.

\section{Statistical analysis}

All data were analyzed using the Statistical Package for the Social Sciences for Windows (SPSS version 15) (Chicago, IL, USA). One way analysis of variance (ANOVA) and Chi-square analysis were used to examine weight change according to selected socio-demographic characteristics.
The general linear model of analysis of covariance (ANCOVA) was used to test the differences in dietary intake, and physical activity by weight change groups with household income, years of education, age at diagnosis and time since diagnosis as covariates. The LSD (Least Significant Difference) test was applied to evaluate the statistically significant mean difference among the weight change groups.

\section{Results}

Table 1 shows the socio-demographic characteristics, reproductive and breast cancer history of respondents. The median age of the women was 53 years. Among the women, more than half $(57.1 \%)$ were Malay, $33.2 \%$ Chinese and $9.8 \%$ Indian. More than half had completed secondary education (51.9\%) and $69.3 \%$ were currently unemployed. Over $80 \%$ of the women were post-menopausal at study entry. The mean duration of time from diagnosis to study entry was 4.86 years (95\% CI: 4.50 5.21 ). About $41.0 \%$ of the women were diagnosed with cancer stage II, $31.8 \%$ stage I and $15.7 \%$ stage III. About $69 \%$ of the women reported a family history of cancer. The type of treatments that the women received varied depending on their stage of cancer at the time of diagnosis. Most women had undergone a mastectomy (79.6\%) and chemotherapy (82.9\%).

Table 2 describes the weight status of respondents. The mean weight a year preceding breast cancer diagnosis, at diagnosis and current weight were $59.36 \mathrm{~kg}(95 \%$ CI: $58.17-60.55), 58.36 \mathrm{~kg}$ (95\% CI: 57.51-59.95) and $62.09 \mathrm{~kg}$ (95\% CI: 60.75-63.43), respectively. Nearly half of the women (49.2\%) had normal BMI a year preceding breast cancer diagnosis. However, the percentage of women with normal BMI decreased to about $41 \%$ at study entry. Nearly two-thirds (65.7\%) of the women were abdominally obese based on waist circumference. The mean weight change and percentage of weight change in women from a year preceding breast cancer diagnosis to study entry were $2.73 \mathrm{~kg}$ (95\% CI: 1.90 3.55 ) and $5.17 \%$ (95\% CI: 3.72-6.61), respectively. About $47.8 \%$ of women gained at least $5 \%$ of weight and about one-third (29.9\%) had gained $>10 \%$ of weight.

Dietary intake and physical activity of women after breast cancer diagnosis are described in Table 3. The mean total energy intake of women was 1,350 kcal/day (95\% CI: 1307.56-1391.84). The mean percentage of energy from carbohydrate, protein and fat were 56.02\% (95\% CI: 54.9857.08), $16.38 \%$ (95\% CI: $15.92-16.86)$ and $27.47 \%$ (95\% CI: 26.61-28.34), respectively. Women reported an average of 6.09 daily servings of grains and cereals, 2.18 daily servings of meats and legumes, 0.76 daily servings of fruits, 1.18 daily servings of vegetables and 0.33 daily servings of dairy products, respectively. Most of the women (92.4\%) did not currently consume alcohol. About $42 \%$ of the respondents 
Table 1 Socio-demographic characteristics, reproductive and breast cancer history of women

\begin{tabular}{l} 
Characteristics \\
\hline Age (years) (Median) \\
Ethnicity \\
Malay \\
Chinese \\
Indian and Punjabi \\
Marital status \\
Single \\
Married \\
Divorced/Widowed \\
Education years (years) \\
Employment status \\
Employed \\
Unemployed \\
Pre-menopausal \\
Ponthly personal income (RM) \\
Monthly household income \\
(RM) \\
entry
\end{tabular}

Age at diagnosis (years)

Time since diagnosis (years)

Stage of cancer

$\begin{array}{ll}\text { Stage I } & 117(31.8) \\ \text { Stage II } & 151(41.0) \\ \text { Stage III } & 58(15.8) \\ \text { Not known } & 42(11.4)\end{array}$

Treatment

$\begin{array}{ll}\text { Lumpectomy } & 62(16.8) \\ \text { Mastectomy } & 293(79.6) \\ \text { Radiotherapy } & 293(79.6) \\ \text { Chemotherapy } & 305(82.9) \\ \text { Hormonal therapy } & 159(43.2)\end{array}$

Family history of cancer

$\begin{array}{ll}\text { Yes } & 115(31.2) \\ \text { No } & 253(68.8)\end{array}$

$636.84(500.32-768.12)$

$48.53(47.60-49.46)$

$4.86(4.50-5.21)$
Table 2 Weight status of women with breast cancer

\begin{tabular}{|c|c|c|}
\hline Weight information & Total & Mean $(95 \% \mathrm{Cl})$ \\
\hline $\begin{array}{l}\text { Weight a year preceding breast cancer } \\
\text { diagnosis }(\mathrm{kg})\end{array}$ & & $59.36(58.17-60.55)$ \\
\hline Weight at time of diagnosis $(\mathrm{kg})$ & & $58.36(57.51-59.95)$ \\
\hline Weight at study entry (kg) & & $62.09(60.75-63.43)$ \\
\hline Height (m) & & $1.55(1.54-1.55)$ \\
\hline
\end{tabular}

Waist circumference at study entry $(\mathrm{cm})^{\mathrm{b}}$

$84.81(83.62-85.99)$

$\begin{array}{ll}\text { Increased risk }(\geq 80) & 94(25.5) \\ \text { Substantial risk }(\geq 88) & 148(40.2)\end{array}$

BMI a year preceding breast cancer diagnosis $\left(\mathrm{kg} / \mathrm{m}^{2}\right)^{\mathrm{a}}$

$24.82(24.35-25.29)$

\begin{tabular}{|c|c|c|}
\hline \multirow[t]{5}{*}{$8.96(8.56-9.36)$} & diagnosis $\left(\mathrm{kg} / \mathrm{m}^{2}\right)^{a}$ & \\
\hline & Underweight $(<18.5)$ & $21(5.7)$ \\
\hline & Normal (18.5-24.9) & $181(49.2)$ \\
\hline & Overweight (25.0-29.9) & $122(33.2)$ \\
\hline & Obese $(\geq 30.0)$ & $44(12.0)$ \\
\hline
\end{tabular}

2022.58 (1897.89-2426.08)

BMI at study entry $\left(\mathrm{kg} / \mathrm{m}^{2}\right)^{\mathrm{a}}$

$25.93(25.41-26.45)$

Underweight $(<18.5)$

$20(5.4)$

Normal (18.5-24.9) $151(41.0)$

Overweight (25.0-29.9) 38 (37.5)

Obese $(\geq 30.0) \quad 59(16.0)$

Weight change from a year preceding diagnosis to study entry $(\mathrm{kg})$
$>10 \mathrm{~kg}$ weight loss
$15(4.1)$
5-10 kg weight loss
$39(10.6)$
$0-<5 \mathrm{~kg}$ weight loss
$83(22.6)$
$105(28.5)$
$71(19.3)$
5-10 kg weight gain
$55(14.9)$
$>10 \mathrm{~kg}$ weight gain

$2.73(1.90-3.55)$

$5.17(3.72-6.61)$

Percentage of weight change from a year preceding diagnosis to study entry (\%)
$>10 \%$ weight loss
$40(10.9)$
$5-10 \%$ weight loss
$41(11.1)$
$0-<5 \%$ weight loss
$54(14.7)$
$0-<5 \%$ weight gain
$57(15.5)$
$5-10 \%$ weight gain
$66(17.9)$
$>10 \%$ weight gain
$110(29.9)$

a World Health Organization, (1998).

${ }^{\mathrm{b}}$ Increased risk of metabolic complications associated with obesity.

c Substantial risk of metabolic complications associated with obesity.

had a high level of activity, 38.3\% moderate and 19.6\% low activity levels. Most respondents (86.1\%) were engaged in work/domestic, transport related (61.4\%) and recreational (41.0\%) activities. 
Table 3 Dietary intake and physical activity of women after breast cancer diagnosis

\begin{tabular}{|c|c|c|}
\hline & Total & Mean $(95 \% \mathrm{Cl})$ \\
\hline \multicolumn{3}{|l|}{ Dietary intake } \\
\hline $\begin{array}{l}\text { Total energy intake } \\
\text { (kcal/day) }\end{array}$ & & $1350(1307.56-1391.84)$ \\
\hline $\begin{array}{l}\text { Percentage of energy from } \\
\text { carbohydrate (\%) }\end{array}$ & & $56.02(54.98-57.08)$ \\
\hline $\begin{array}{l}\text { Percentage of energy from } \\
\text { protein (\%) }\end{array}$ & & $16.38(15.92-16.86)$ \\
\hline $\begin{array}{l}\text { Percentage of energy from } \\
\text { fat }(\%)\end{array}$ & & $27.47(26.61-28.34)$ \\
\hline $\begin{array}{l}\text { Number of servings from } \\
\text { grains and cereals }\end{array}$ & & $6.09(5.91-6.35)$ \\
\hline $\begin{array}{l}\text { Number of servings from } \\
\text { meats } b\end{array}$ & & $2.18(2.04-2.29)$ \\
\hline $\begin{array}{l}\text { Number of servings from } \\
\text { fruits }\end{array}$ & & $0.76(0.61-0.82)$ \\
\hline $\begin{array}{l}\text { Number of serving from } \\
\text { vegetables }\end{array}$ & & $1.18(1.08-1.24)$ \\
\hline $\begin{array}{l}\text { Number of serving from } \\
\text { dairy products }{ }^{c}\end{array}$ & & $0.33(0.27-0.39)$ \\
\hline \multicolumn{3}{|l|}{ Alcohol intake } \\
\hline Yes & $28(7.6)$ & \\
\hline No & $340(92.4)$ & \\
\hline
\end{tabular}

Physical activity

Total physical activity

(MET-min/week)

Low

Moderate

High

Activities at work/domestic (min/day)

Did work activity $\quad 317$ (86.1)

No work activity

Travel to and from places (min/day)

Did travel activity

$226(61.4)$

No travel activity

$142(38.6)$

Recreational activities

(min/day)

Did recreational activity 151 (41.0)

No recreational activity 169 (45.9)

Sedentary behavior ${ }^{d}$ (min/day)
Among socio-demographic and cancer characteristics of women, only BMI a year before diagnosis was significantly different by weight change groups (Table 4). Women with weight gains have significantly lower prediagnosis BMI than those with weight loss or stable weight (Table 4). Dietary intake and physical activity of women by weight changes are presented in Table 5. The number of servings for fruits and vegetables and dairy products differed significantly among the weight change groups. The least number of servings for fruits and vegetables was reported for the $>10 \%$ weight gain group (1.58 servings/day; 95\% CI: $1.36-1.82)$ compared to other weight change groups. In contrast, the $>10 \%$ weight gain group was found to have the greatest number of servings for dairy products compared to the $>10 \%$ weight loss group $(\mathrm{p}<0.05)$, although none of the groups achieved the recommended 2-3 servings/ day of milk and dairy products. Women in all weight change groups did not differ significantly in physical activity.

\section{Discussion}

Weight gain is a common observation among women with breast cancer. This study supports the existing evidence that women tend to gain weight after breast cancer diagnosis. A significant gain in weight amounting to $2-3 \mathrm{~kg}$ after diagnosis of breast cancer was found in this study. Although some aspects of weight gain pattern among the breast cancer survivors in our study were similar to those observed in other studies $[12,27,28]$, the magnitude of weight gain differed. The magnitude of weight gain observed from pre-diagnosis to study entry was somewhat similar $(2.4 \mathrm{~kg})$ to that reported by Caan et al., (2006) [17], but was lower $(3.17 \mathrm{~kg})$ than that reported in the WHEL study [29]. In both studies, the mean duration between time of diagnosis and study entry was similar (4-5 years) to our study.

Overweight and obesity were already prevalent in over $40 \%$ of the women prior to diagnosis, however more women were overweight and obese at study entry. Similar to Rock et al. (1999) [7], we also showed that women with higher weight gains had significantly lower pre-diagnosis BMI than those with lower weight gains and stable weight. A higher body mass index (BMI) in women after breast cancer diagnosis will increase the risk for recurrence and lower the survival $[7,12]$. Kroenke et al. (2005) [30] showed that breast cancer survivors who increased their body mass index by $0.5-2.0 \mathrm{~kg} / \mathrm{m}^{2}$ had a risk ratio of recurrence of 1.4 and those who gained more than $2.0 \mathrm{~kg} / \mathrm{m}^{2}$ had a risk ratio of 1.53 , with both groups having significantly higher allcause mortality compared to survivors with a stable weight. The clinical significance of weight gain $(2.7 \mathrm{~kg})$ observed in the present study is not known, however

\footnotetext{
${ }^{a}$ grains and cereals-grain/cereal/tubers based on Malaysian Dietary Guidelines (2010).

${ }^{b}$ meats-meat/fish/poultry/legumes based on Malaysian Dietary Guidelines (2010).

c dairy products-milk/dairy products based on Malaysian Dietary Guidelines (2010).

d Sitting or reclining at work, at home, getting to and from places including time spent on sitting at desk, travelling in car, bus or watching television but do not include time spent sleeping.
} 
Table 4 Socio-demographic and cancer characteristics of women by weight change groups

\begin{tabular}{|c|c|c|c|c|c|c|}
\hline \multirow[t]{2}{*}{ Characteristics } & \multicolumn{3}{|c|}{ Weight changes groups since diagnosis } & \multicolumn{2}{|l|}{ Mean $(95 \% \mathrm{Cl})$} & \multirow[t]{2}{*}{ p-value } \\
\hline & $\begin{array}{l}>10 \% \\
\text { weight loss } \\
(n=40)\end{array}$ & $\begin{array}{l}5-10 \% \\
\text { weight loss } \\
(n=41)\end{array}$ & $\begin{array}{l}\text { No change* } \\
(n=114)\end{array}$ & $\begin{array}{l}5-10 \% \\
\text { weight gain } \\
(n=60)\end{array}$ & $\begin{array}{l}>10 \% \\
\text { weight gain } \\
(n=113)\end{array}$ & \\
\hline Age at diagnosis (years) & $\begin{array}{l}50.05 \\
(47.45-52.65)\end{array}$ & $\begin{array}{l}51.02 \\
(48.17-53.87)\end{array}$ & $\begin{array}{l}49.19 \\
(47.34-51.05)\end{array}$ & $\begin{array}{l}47.28 \\
(45.11-49.46)\end{array}$ & $\begin{array}{l}47.05 \\
(45.47-48.63)\end{array}$ & 0.06 \\
\hline \multicolumn{7}{|l|}{ Ethnicity } \\
\hline Malay & $24(58.5)$ & $20(48.8)$ & $60(52.6)$ & $34(56.7)$ & $72(19.6)$ & 0.34 \\
\hline Non-Malay & $17(41.5)$ & $21(51.2)$ & $54(47.4)$ & $26(43.3)$ & $40(35.7)$ & \\
\hline Marital status & & & & & & 0.43 \\
\hline Married & $35(85.4)$ & $36(87.8)$ & $90(78.9)$ & $53(88.3)$ & $90(80.4)$ & \\
\hline Others** & $6(14.6)$ & $5(12.2)$ & $24(21.1)$ & $7(11.7)$ & $22(19.6)$ & \\
\hline Education (years) & $\begin{array}{l}8.56 \\
(7.26-9.86)\end{array}$ & $\begin{array}{l}8.54 \\
(7.30-9.78)\end{array}$ & $\begin{array}{l}8.68 \\
(7.93-9.44)\end{array}$ & $\begin{array}{l}9.92 \\
(8.93-10.90)\end{array}$ & $\begin{array}{l}9.03 \\
(8.34-9.71)\end{array}$ & 0.28 \\
\hline Secondary and lower & $22(53.7)$ & $24(58.5)$ & $58(50.9)$ & $22(36.7)$ & $58(51.8)$ & \\
\hline Tertiary and others & $19(46.3)$ & $17(41.5)$ & $56(49.1)$ & $38(63.3)$ & $54(48.2)$ & \\
\hline Employment status & & & & & & 0.86 \\
\hline Employed & $10(24.4)$ & $12(29.3)$ & $34(29.8)$ & $20(33.3)$ & $37(33.0)$ & \\
\hline Unemployed & $31(75.6)$ & $29(70.7)$ & $80(70.2)$ & $40(66.7)$ & $75(67.0)$ & \\
\hline Monthly household income (RM) & $\begin{array}{l}1756.10 \\
(1246.58-2265.61)\end{array}$ & $\begin{array}{l}2002.63 \\
(1252.71-2752.56)\end{array}$ & $\begin{array}{l}1967.76 \\
(1563.28-2372.25)\end{array}$ & $\begin{array}{l}2968.67 \\
(2089.90-3847.44)\end{array}$ & $\begin{array}{l}2134.45 \\
(1620.90-2647.99)\end{array}$ & 0.10 \\
\hline Menopausal status at study entry & & & & & & 0.10 \\
\hline Pre-menopausal & $3(7.3)$ & $4(9.8)$ & $24(21.1)$ & $14(23.3)$ & $25(22.3)$ & \\
\hline Post-menopausal & $38(92.7)$ & $37(90.2)$ & $90(78.9)$ & $46(76.7)$ & $87(77.7)$ & \\
\hline Time since diagnosis (years) & $\begin{array}{l}4.63 \\
(3.41-5.86)\end{array}$ & $\begin{array}{l}5.20 \\
(3.74-6.65)\end{array}$ & $\begin{array}{l}4.67 \\
(4.03-5.31)\end{array}$ & $\begin{array}{l}4.48 \\
(3.69-5.28)\end{array}$ & $\begin{array}{l}5.21 \\
(4.65-5.78)\end{array}$ & 0.59 \\
\hline Stage of cancer & & & & & & 0.89 \\
\hline Stage I & $9(24.3)$ & $12(31.6)$ & $36(37.5)$ & $22(40.7)$ & $38(37.6)$ & \\
\hline Stage ॥ & $21(56.8)$ & $19(50.0)$ & $43(44.8)$ & $22(40.7)$ & $46(45.5)$ & \\
\hline Stage III & $7(18.9)$ & $7(18.4)$ & $17(17.7)$ & $10(18.5)$ & $17(16.8)$ & \\
\hline $\begin{array}{l}\text { BMl a year preceding diagnosis*** } \\
\left(\mathrm{kg} / \mathrm{m}^{2}\right)\end{array}$ & $\begin{array}{l}26.62 \\
(24.86-28.37)\end{array}$ & $\begin{array}{l}25.96 \\
(24.45-27.47)\end{array}$ & $\begin{array}{l}25.18 \\
(24.33-26.04)\end{array}$ & $\begin{array}{l}24.50 \\
(23.38-25.62)\end{array}$ & $\begin{array}{l}23.54 \\
(22.78-24.29)\end{array}$ & $0.001^{* * *}$ \\
\hline
\end{tabular}

${ }^{*} 0-5 \%$ weight loss or weight gain.

**Single, widowed or divorced.

***Body mass index a year preceding breast cancer diagnosis.

**** $p<0.05$.

${ }^{\text {a }}$ A significant difference between $>10 \%$ weight loss and $5-10 \%$ weight gain groups, at $\mathrm{p}<0.05$.

${ }^{b}$ A significant difference between $>10 \%$ weight loss and $>10 \%$ weight gain groups, at $p<0.05$.

c A significant difference between $5-10 \%$ weight loss and $>10 \%$ weight gain groups, at $p<0.05$.

${ }^{d}$ A significant difference between no change and $>10 \%$ weight gain groups, at $p<0.05$.

the calculated increase (1 year before diagnosis to study entry) in body mass index is $1.11 \mathrm{~kg} / \mathrm{m}^{2}$ which if based on previous studies could have significant effect on cancer recurrence and survival. About two-thirds of the women (65.8\%) in this present study had at risk waist circumference. Having a higher BMI and waist circumference could put these women at risk of cardiovascular diseases, diabetes and cancer recurrence [31].

We found that women who gained weight after breast cancer diagnosis had lower fruit and vegetable intakes compared to those who lost weight. Although the weight loss groups had a higher intake of fruit and 
Table 5 Lifestyle behaviors of women by weight change groups

\begin{tabular}{|c|c|c|c|c|c|c|}
\hline & & $\begin{array}{l}\text { Weight change since } \\
\text { diagnosis }\end{array}$ & $\begin{array}{l}\text { Mean }(95 \% \\
\text { Cl) }\end{array}$ & & & p-value \\
\hline & $\begin{array}{l}>10 \% \\
\text { weight loss } \\
(n=40)\end{array}$ & $\begin{array}{l}5-10 \% \\
\text { weight loss } \\
(\mathrm{n}=41)\end{array}$ & $\begin{array}{l}\text { No change* }^{*} \\
(n=114)\end{array}$ & $\begin{array}{l}5-10 \% \\
\text { weight gain } \\
(n=60)\end{array}$ & $\begin{array}{l}>10 \% \\
\text { weight gain } \\
(n=113)\end{array}$ & \\
\hline Total energy intake (kcal/day) & 1344 & 1408 & 1330 & 1377 & 1335 & 0.82 \\
\hline $\begin{array}{l}\text { Percentage of energy intake from } \\
\text { carbohydrate (\%) }\end{array}$ & $\begin{array}{l}55.76 \\
(52.66-59.03)\end{array}$ & $\begin{array}{l}55.20 \\
(52.00-58.39)\end{array}$ & $\begin{array}{l}56.79 \\
(54.88-58.70)\end{array}$ & $\begin{array}{l}55.73 \\
(53.08-58.39)\end{array}$ & $\begin{array}{l}55.81 \\
(53.85-57.72)\end{array}$ & 0.90 \\
\hline $\begin{array}{l}\text { Percentage of energy intake from protein } \\
\text { (\%) }\end{array}$ & $\begin{array}{l}16.15 \\
(14.62-17.45)\end{array}$ & $\begin{array}{l}17.41 \\
(15.99-18.83)\end{array}$ & $\begin{array}{l}15.76 \\
(14.91-16.61)\end{array}$ & $\begin{array}{l}16.66 \\
(15.48-17.84)\end{array}$ & $\begin{array}{l}16.59 \\
(15.77-17.49)\end{array}$ & 0.30 \\
\hline Percentage of energy intake from fat (\%) & $\begin{array}{l}28.10 \\
(25.52-30.75)\end{array}$ & $\begin{array}{l}27.35 \\
(24.73-29.97)\end{array}$ & $\begin{array}{l}27.50 \\
(25.93-29.07)\end{array}$ & $\begin{array}{l}27.49 \\
(25.31-29.67)\end{array}$ & $\begin{array}{l}27.27 \\
(25.66-28.83)\end{array}$ & 0.98 \\
\hline $\begin{array}{l}\text { Number of servings from grains and } \\
\text { cereals }\end{array}$ & $\begin{array}{l}5.99 \\
(5.40-6.70)\end{array}$ & $\begin{array}{l}6.88 \\
(6.23-7.54)\end{array}$ & $\begin{array}{l}6.23 \\
(5.84-6.62)\end{array}$ & $\begin{array}{l}5.70 \\
(5.15-6.24)\end{array}$ & $\begin{array}{l}6.04 \\
(5.62-6.41)\end{array}$ & 0.87 \\
\hline Number of servings from meats & $\begin{array}{l}2.08 \\
(1.72-2.45)\end{array}$ & $\begin{array}{l}2.49 \\
(2.13-2.85)\end{array}$ & $\begin{array}{l}2.12 \\
(1.91-2.34)\end{array}$ & $\begin{array}{l}2.13 \\
(1.83-2.44)\end{array}$ & $\begin{array}{l}2.16 \\
(1.94-2.38)\end{array}$ & 0.49 \\
\hline $\begin{array}{l}\text { Number of servings from fruits and } \\
\text { vegetables }\end{array}$ & $\begin{array}{l}2.06 \\
(1.66-2.41)\end{array}$ & $\begin{array}{l}2.12 \\
(1.74-2.50)\end{array}$ & $\begin{array}{l}2.00 \\
(1.77-2.22)\end{array}$ & $\begin{array}{l}1.94 \\
(1.62-2.26)\end{array}$ & $\begin{array}{l}1.58 \\
(1.36-1.82)\end{array}$ & ${ }_{c}^{0.04^{* * a,} b,}$ \\
\hline Number of serving from dairy products & $\begin{array}{l}0.19 \\
(0.01-0.36)\end{array}$ & $\begin{array}{l}0.31 \\
(0.13-0.49)\end{array}$ & $\begin{array}{l}0.33 \\
(0.22-0.44)\end{array}$ & $\begin{array}{l}0.31 \\
(0.17-0.46)\end{array}$ & $\begin{array}{l}0.41 \\
(0.30-0.52)\end{array}$ & $0.03^{* * a}$ \\
\hline Alcohol intake & & & & & & 0.12 \\
\hline Yes & $2(7.1)$ & $1(3.6)$ & $12(42.9)$ & $6(21.4)$ & $7(25.0)$ & \\
\hline No & $38(11.2)$ & $40(11.8)$ & $102(30.0)$ & $54(15.9)$ & $106(31.2)$ & \\
\hline Total physical activities (MET-min/week) & $\begin{array}{l}4436.71 \\
(3112.63 \\
5602.65)\end{array}$ & $\begin{array}{l}4246.85 \\
(2996.98,5494.10)\end{array}$ & $\begin{array}{l}2817.77 \\
(2071.49 \\
3563.63)\end{array}$ & $\begin{array}{l}3753.38 \\
(2717.08 \\
4790.58)\end{array}$ & $\begin{array}{l}3351.85 \\
(2616.35 \\
4126.77)\end{array}$ & 0.15 \\
\hline Low & $5(12.5)$ & $9(22.0)$ & $28(24.6)$ & $12(20.0)$ & $18(15.9)$ & 0.16 \\
\hline Moderate & $16(40.0)$ & $11(26.8)$ & $47(41.2)$ & $17(28.3)$ & $50(44.2)$ & \\
\hline High & $19(47.5)$ & $21(51.2)$ & $39(34.2)$ & $31(51.7)$ & $45(39.8)$ & \\
\hline
\end{tabular}

Adjusted for household income, years of education, age at diagnosis and time since diagnosis.

* $0-5 \%$ weight loss or weight gain.

** $\mathrm{p}<0.05$.

a A significant difference between $>10 \%$ weight loss and $>10 \%$ weight gain groups, at $p<0.05$.

${ }^{b}$ A significant difference between $5-10 \%$ weight loss and $>10 \%$ weight gain groups, at $p<0.05$.

${ }^{c}$ A significant difference between no change and $>10 \%$ weight gain groups, at $p<0.05$.

vegetables, the number of servings still did not meet the recommendation of 3-5 servings daily. Increasing the consumption of fruits and vegetables may reduce the risk of overweight/obesity as well as facilitate the slowing down of weight gain, especially among overweight and obese adults [32]. However, the Women's Healthy Eating and Living (WHEL) study, a randomized controlled dietary intervention study that examined the effect of plant-based diets without a specific energy restriction among breast cancer survivors, showed that diets high in fruits, vegetables and fiber were not significantly associated with weight change [33]. Although the mechanism underlying fruit and vegetable intake and body weight is still uncertain, it is known that fruits and vegetables are generally low in energy density, which could influence energy intake [34]. Consumption of $\geq 9$ servings of fruit and vegetables is associated with lower energy density as well as a lower prevalence of obesity even for diets high in fat [35]. Furthermore, fruits and vegetables have a high water and fiber content, which, in turn, promotes satiety. Therefore, women who consume more fruits and vegetables are more likely to lose weight compared to those who consume less fruit and vegetables.

Adequate consumption of dairy products is encouraged because they are rich sources of essential nutrients that are important for bone health. The present study found that women who gained $>10 \%$ of weight consumed significantly more servings of dairy products than those who lost $>10 \%$ of weight after breast cancer diagnosis; although none of the groups met the recommended intake of 1-3 servings of dairy products. A plausible explanation for the low dairy product intake among these women is that many believed milk and dairy products are high in saturated fat and cholesterol, and contain carcinogens that can cause cancer recurrence. Although we 
did not examine changes in dietary intake before and after cancer diagnosis, it is possible that these women could have stopped or reduced the intake of dairy products since cancer diagnosis. Skeie et al. (2009) [36] also reported that breast cancer survivors reduced their milk consumption after breast cancer diagnosis. However, the Women's Intervention Nutrition Study (WINS) showed that breast cancer survivors had 1.6-1.8 servings per day of dairy products [37]. Several studies [38,39] have reported that low fat dairy products could prevent weight gain and promote weight loss among healthy adult population. An adequate dairy consumption in conjunction with controlled/reduced caloric intake may be associated with weight regulation [39-41] and reduced adiposity [42,43], indicating that dairy intake may be valuable for weight maintenance.

Most of the women (80.4\%) in the present study had moderate and high physical activity level. This physical activity pattern is consistent with the findings of a study among United States women that showed $70 \%$ of women with breast cancer had moderate-intensity physical activity while $10 \%$ had high-intensity physical activity [19]. However, another study [44] among breast cancer survivors found that the majority $(74.6 \%)$ had low intensity physical activity and only $25.4 \%$ had moderate and high intensity physical activity. In addition, cancer survivors have also been reported to be more active than non-cancer controls [45]. Perhaps, they are more likely to adopt healthier lifestyle changes [46], which could explain why most of the women in the present study had at least a moderate-intensity physical activity.

This study has several limitations. The small sample size of this study may not be representative of the population of women with breast cancer in Malaysia. Despite the recruitment of respondents from hospitals and breast cancer support groups throughout the country, there could still be differences in socio-demographic, reproductive and other characteristics. Another limitation is that study participation was voluntary which could result in selection bias in that those who are weight or health conscious would participate in the study. This study used self-reported weight a year preceding breast cancer diagnosis, which is subject to recall error and consequently could affect the calculation and classification of weight change. However, self-reported weight has been shown to be accurate [47] and most epidemiological studies have used self-reported weight and weight change data $[48,49]$. Self-reported measures also tend to overestimate desirable health behaviors such as physical activity and intake of fruits and vegetables. Being overweight and diagnosed with breast cancer may prompt women to over-report healthy behaviors. In contrast, undesirable health behaviors (i.e. smoking, sedentary lifestyle, high dietary fat intake) will be underreported. This study was cross-sectional in that the information on factors associated with weight change could not imply cause and effect relationship. A longitudinal study design is most appropriate to identify changes in lifestyle behaviors that cause weight loss or gain in cancer survivors. As we did not measure weight, dietary intake and physical activity level at other times (e.g. every year after breast cancer diagnosis), the selection of period to measure these variables could contribute to different results. For example, it is possible that although the study only measured weight status at fixed periods, i.e. a year before breast cancer diagnosis, at diagnosis and at study entry, women will initially lose or gain weight at different times. It is also possible that women make lifestyle changes from time to time and that their current diets or physical activity may be better or healthier compared to their earlier practices. Despite these limitations, this is the first study to investigate weight change before and after diagnosis in a sample of Malaysian women diagnosed with breast cancer.

\section{Conclusion}

The high proportion of breast cancer survivors experiencing weight gain in this study suggests that efforts are needed to encourage breast cancer survivors to achieve or maintain healthy body weight. Understanding lifestyle behaviors i.e. dietary intake and physical activity of women diagnosed with breast cancer and with varying degrees of weight change is essential for the development and targeting of effective strategies to improve health and survival of breast cancer survivors. As the intake of fruits, vegetables and dairy products of women in this study are still below the recommended servings per day, dietary strategies should target all breast cancer survivors, regardless of their weight status. Despite limited evidence on how lifestyle behaviors prevents cancer recurrence or prolongs life, a diagnosis of breast cancer should be a starting point for women to have healthful diets and regular physical activity.

\section{Acknowledgements}

This research was supported by funding from the Fundamental Research Grant Scheme (FRGS) of Universiti Putra Malaysia. The authors acknowledge the assistance of all hospitals and breast cancer support groups and participation of breast cancer survivors in the study.

\section{Author details}

'Department of Nutrition and Dietetics, Faculty of Medicine and Health Sciences, Universiti Putra Malaysia, Serdang 43400 Selangor, Malaysia. ${ }^{2}$ Cancer Education \& Services Laboratary, Institute of Social Science Studies, Universiti Putra Malaysia, Serdang 43400 Selangor, Malaysia. ${ }^{3}$ Department of Radiotherapy and Oncology, Hospital Kuala Lumpur, 50586 Kuala Lumpur, Malaysia. ${ }^{4}$ Department of Community Health, Faculty of Medicine and Health Sciences, Universiti Putra Malaysia, Serdang 43400 Selangor, Malaysia.

\section{Authors' contributions}

YHY designed the study, conducted data collection, performed and interpreted the analyses and prepared the manuscript; ZMS designed the study, supervised and assisted data collection, analysed and interpreted of study findings, assisted in the preparation of the manuscript and approved 
the final manuscript; CYM, ZH, MK, RMY, ZO, NS and YHW supervised and assisted in study design and data collection. All authors read and revised the manuscript.

\section{Competing interests}

The authors declare that they have no competing interests.

Received: 17 August 2010 Accepted: 13 May 2011

Published: 13 May 2011

\section{References}

1. World Health Organization: World Health Statistics 2008 Geneva: World Health Organization; 2008.

2. Lim GCC, Halimah Y, Lim TO: First Report of the National Cancer Registry: Cancer Incidence in Malaysia 2002 Kuala Lumpur: National Cancer Registry; 2003.

3. Zainal AO, Zainudin MA, Nor Saleha IT: Malaysian Cancer Statistics-Data and Figure Peninsular Malaysia 2006 National Cancer Registry. Ministry of Health Malaysia: Kuala Lumpur; 2006

4. Sant M, Aareleid T, Berrino F, Lasota MB, Carli PM, Faivre J, Grosclaude P, Hedelin G, Matsuda T, Mollers H, Moller T, Verdecchia A, Capocaccia R, Gatta G, Micheli A, Santaquilani M, Roazzi P, Lisi D, EUROCARE Working Group: EUROCARE-3: survival of cancer patients diagnosed 1990-94results and commentary. Annals of Oncology 2003, 14(5):v61-v118.

5. American Cancer Society (ACS): Breast Cancer Facts \& Figures 2007-2008 American Cancer Society, Atlanta, Georgia; 2007.

6. Yip $\mathrm{CH}$, Nur Aishah MT, Ibraham M: Epidemiology of breast cancer in Malaysia. Asian Pacific Journal of Cancer Prevention 2006, 7:369-374.

7. Rock CL, Flatt SW, Newman V, Caan BJ, Haan MN, Stefanick ML, Faerber S, Pierce JP: Factors associated with weight gain in women after diagnosis of breast cancer. Journal of the American Dietetic Association 1999, 99(10):1212-1221.

8. Halbert CH, Weathers B, Esteve R, Audrain-McGovern J, Kumanyika S, DeMichele A, Barg F: Experiences with weight change in AfricanAmerican breast cancer survivors. The Breast Journal 2008, 14(2):182-187.

9. Heideman WH, Russell NS, Gundy C, Rookus MA, Voskuil DW: The frequency, magnitude and timing of post-diagnosis body weight gain in Dutch breast cancer survivors. European Journal of Cancer 2009, 45(1):119-126.

10. Makari-Judson G, Judson CH, Mertens WC: Longitudinal patterns of weight gain after breast cancer diagnosis: Observations beyond the first year. The Breast Journal 2007, 13(3):258-265.

11. Rock CL, Demark-Wahnefried W: Nutrition and survival after the diagnosis of breast cancer: a review of the evidence. Journal of Clinical Oncology 2002, 20:3302-3306.

12. Irwin $M L$, McTiernan $A$, Baumgartner RN, Baumgartner KB, Bernstein $L$, Gilliland FD, Ballard-Barbash R: Changes in body fat and weight after a breast cancer diagnosis: Influence of demographic, prognostic, and lifestyle factors. Journal of Clinical Oncology 2005, 23(4):774-782.

13. Salminen EK, Lagstrom HK, Heikkila SP, Salminen SJ: Does breast cancer patients' change dietary habits? European Journal of Clinical Nutrition 2000, 54:844-848.

14. Maunsell E, Drolet M, Brisson J, Robert J, Deschenes L: Dietary chang after breast cancer: extent, predictors, and relation with psychological distress. American Society of Clinical Oncology 2002, 20:1017-1025.

15. Thomson CA, Flatt SW, Rock CL, Ritenbaugh C, Newman VA, Pierce JP: Increased fruit, vegetable and fiber intake and lower fat intake reported among women previously treated for invasive breast cancer. Journal of the American Dietetic Association 2002, 102:801-808.

16. Wayne SJ, Lopez ST, Butler LM, Baumgartner KB, Baumgartner RN, BallardBarbash R: Changes in Dietary Intake after Diagnosis of Breast Cancer. Journal of the American Dietetic Association 2004, 104:1561-1568.

17. Irwin ML, MCTiernan A, Bernstein L, Gilliland FD, Baumgartner R, Baumgartner K, Ballard-Barbash R: Physical activity levels among breast cancer survivors. Medical \& Science in Sports \& Exercise 2004, 36:1484-1491.

18. Harrison S, Hayes SC, Newman B: Level of physical activity and characteristics associated with change following breast cancer diagnosis and treatment. Psycho-Oncology 2009, 18(4):387-394.

19. Emaus A, Veierod MB, Tretli S, Finstad SE, Selmer R, Furberg AS, Bernstein L, Thune I: Metabolic profile, physical activity, and mortality in reast cancer patients. Breast Cancer Research Treatment 2010, 121(3):651-660.
20. First Data Bank: Nutritionist Pro. San Bruno, CA 94066; 2005

21. Tee ES, Ismail MN, Nasir MA, Khatijah I: Nutrient Composition of Malaysia Foods. Kuala Lumpur: Institute of Medical Research; 1997.

22. Department of Nutrition, Ministry of Health: Singapore food facts Singapore 1999.

23. Puwastien P, Burlingame B, Raroengwichit, Sungpuag P: ASEAN Food Composition Tables 2000 Institute of Nutrition: Mahidol University, Thailand; 2000.

24. Global Physical Activity Questionnaire (GPAQ). [http://www.who.int/chp/ steps/GPAQ/en/].

25. World Health Organization (WHO): Physical status: the use and interpretation of anthropometry Report of a WHO Expert Committee. WHO Technical Report Series 854. Geneva; 1995.

26. Doyle C, Kushi LH, Byers T: Nutrition and physical activity during and after cancer treatment: an American Cancer Society guide for informed choices. Cancer Journal for Clinicians 2006, 56(6):323-353.

27. Camoriano J, Loprinzi C, Ingle J, Therneau T, Krook J, Veeder M: Weight change in women treated with adjuvant therapy or observed following mastectomy for node-positive breast cancer. Journal of Clinical Oncology 1990, 8:1327-1334.

28. Gu K, Chen Xl, Zheng Y, Chen Z, Zheng W, Lu W, Shu XO: Weight change patterns among breast cancer survivors: results from the Shanghai breast cancer survival study. Cancer Causes and Control 2009, 21(4):621-629.

29. Pierce JP, Faerber S, Wright FA, Rock CL, Newman V, Flatt SW, Kealey S, Jones VE, Caan BJ, Gold EB, Haan M, Hollenbach KA, Jones L, Marshall JR, Ritenbaugh C, Stefanick ML, Thomson C, Wasserman L, Natarajan L, Thomas RG, Gilpin EA, the Women's Healthy Eating and Living (WHEL) Study Group: A randomized trial of the effect of a plant-based dietary pattern on additional breast cancer events and survival: the Women's Healthy Eating and Living (WHEL) Study. Controlled Clinical Trials 2002, 23(6):728-756.

30. Kroenke $\mathrm{CH}$, Chen WY, Rosner B, Holmes MD: Weight, weight gain, and survival after breast cancer diagnosis. Journal of Clinical Oncology 2005, 23:1370-1378.

31. Caan BJ, Kwan ML, Hartzell G, Castillo A, Slattery ML, Sternfeld B, Weltzien E: Pre-diagnosis body mass index, post-diagnosis weight and prognosis among women with early stage breast cancer. Cancer Causes Control 2008, 19:1319-1328.

32. Sartorelli DS, Franco LJ, Cardoso MA: High intake of fruits and vegetables predicts weight loss in Brazilian overweight adults. Nutrition Research 2008, 28:233-238.

33. Demark-Wahnefried W, Hars V, Conaway MR, Havlin K, Rimer BK McElveen G, Winer EP: Reduced rates of metabolism and decreased physical activity in breast cancer patients receiving adjuvant chemotherapy. American Journal of Clinical Nutrition 1997, 65:1495-1501.

34. Bell EA, Castellanos VH, Pelkman CL, Thorwart ML, Rolls BJ: Energy density of foods affects energy intake in normal-weight women. American Journal of Clinical Nutrition 1998, 67:412-420.

35. Ledikwe JH, Blanck HM, Khan LK, Serdula MK, Seymour JD, Tohill DC, Rolls BJ: Dietary energy density is associated with energy intake and weight status in US adults. American Journal of Clinical Nutrition 2006, 83(6):1362-1368.

36. Skeie G, Hjartaker A, Braaten T, Lund E: Dietary change among breast and colorectal cancer survivors and cancer-free women in the Norwegian women and cancer cohort study. Cancer Causes Control 2009, 20:1955-1966.

37. Chlebowski RT, Blackburn GL, Thomson CA, Nixon DW, Shapiro A, Hoy MK Goodman MT, Giuliano AE, Karanja N, McAndrew P, Butler CHJ, Merkel D, Kristal A, Caan B, Michaelson R, Vinciguerra V, Prete S, Winkler M, Hall R, Simon M, Winters BL, Elashoff RM: Dietary fat reduction and breast cancer outcome: Interim Efficacy results from the women's intervention nutrition study. Journal of the National Cancer Institute 2006, 98(24):1769-1776.

38. Lau EMC, Woo J, Lam V, Hong A: Milk supplementation of the diet by ostmenopausal Chinese women on a low calcium intake retards bone loss. Journal of Bone and Mineral Research 2001, 16(9):1704-1709.

39. Brooks BM, Rajeshwari R, Nicklas TA, Yang SJ, Berenson GS: Association of calcium intake, dairy product consumption with overweight status in young adults (1995-1996): The Bogalusa Heart Study. Journal of the American College of Nutrition 2006, 25(6):523-532. 
40. Pereira MA, Jacobs DR, Van Horn L, Slattery ML, Kartashov Al, Ludwig DS: Dairy consumption, obesity, and the insulin resistance syndrome in young adults: The CARDIA Study. The Journal of the American Medical Association 2002, 287:2081-2089.

41. Mirmiran P, Esmaillzadeh A, Azizi F: Dairy consumption and body mass index: An inverse relationship. International Journal of Obesity (Lond) 2005, 29:115-121.

42. Zemel MB, Richards J, Mathis S, Milstead A, Gebhardt L, Silva E: Dairy augmentation of total and central fat loss in obese subjects. International Journal of Obesity and Related Metabolic Disorders 2005, 29:391-397.

43. Zemel MB, Richards J, Milstead A, Campbell P: Effects of calcium and dairy on body composition and weight loss in African-American adults. Obesity Research 2005, 13:1218-1225.

44. Abdul-Samad AA, Al-Kamil EA, Al-Sodani AH: Breast Cancer and Selected Lifestyle Variables: A Case-Control Study. Bahrain Medical Bulletin 2009, 31(4):1-10.

45. Bellizi KM, Rowland JH, Jeffery DD, McNeel T: Health Behaviors of Cancers Survivors: Examining Opportunities for Cancer Control Intervention. Journal of Clinical Oncology 2005, 23:8884-8893.

46. Parkin DM, Bray F, Ferlay J, Pisani P: Global cancer statistics, 2002. Cancer Journal for Clinicians 2005, 55:74-108.

47. Spencer EA, Appleby PN, Davey GK, Key TJ: Validity of self-reported height and weight in 4808 EPIC-Oxford participants. Public Health Nutrition 2002, 5:561-565.

48. Loi S, Milne RL, Friedlander ML, McCredie MRE, Giles GG, Hopper JL, Phillips KA: Obesity and outcomes in premenopausal and postmenopausal breast cancer. Cancer Epidemiology, Biomarkers \& Prevention 2005, 14(7):1686-1691.

49. Whiteman MK, Hillis SD, Curtis KM, McDonal JA, Wingo PA, Marchbanks PA: Body mass and mortality after breast cancer diagnosis. Cancer Epidemiology, Biomarkers \& Prevention 2009, 14(8):2009-2014.

\section{Pre-publication history}

The pre-publication history for this paper can be accessed here: http://www.biomedcentral.com/1471-2458/11/309/prepub

doi:10.1186/1471-2458-11-309

Cite this article as: Yaw et al: Weight changes and lifestyle behaviors in women after breast cancer diagnosis: a cross-sectional study. BMC Public Health 2011 11:309.

\section{Submit your next manuscript to BioMed Central and take full advantage of:}

- Convenient online submission

- Thorough peer review

- No space constraints or color figure charges

- Immediate publication on acceptance

- Inclusion in PubMed, CAS, Scopus and Google Scholar

- Research which is freely available for redistribution

Submit your manuscript at www.biomedcentral.com/submit
Biomed Central 